\title{
A FORMULA FOR THE CONDUCTOR OF A SEMIMODULE OF A NUMERICAL SEMIGROUP WITH TWO GENERATORS
}

\author{
PATRICIO ALMIRÓN AND JULIO-JOSÉ MOYANO-FERNÁNDEZ \\ Dedicated to the memory of Fernando Eduardo Torres Orihuela
}

\begin{abstract}
We provide an expression for the conductor $c(\Delta)$ of a semimodule $\Delta$ of a numerical semigroup $\Gamma$ with two generators in terms of the syzygy module of $\Delta$ and the generators of the semigroup. In particular, we deduce that the difference between the conductor of the semimodule and the conductor of the semigroup is an element of $\Gamma$, as well as a formula for $c(\Delta)$ in terms of the dual semimodule of $\Delta$.
\end{abstract}

\section{INTRODUCTION}

A classical problem in the combinatorics of natural numbers is to find a closed expression for the largest natural number that is not representable as a nonnegative linear combination of some relatively prime numbers, called the Frobenius number. This problem can be encoded in terms of getting a formula for the conductor of a numerical semigroup; it is known under the name "Frobenius problem".

Consider $\mathbb{N}=\{x \in \mathbb{Z}: x \geq 0\}$. A numerical semigroup $\Gamma$ is an additive sub-monoid of the monoid $(\mathbb{N},+)$ such that the greatest common divisor of all its elements is equal to 1 . The complement $\mathbb{N} \backslash \Gamma$ is therefore finite, and its elements are called gaps of $\Gamma$. Moreover, $\Gamma$ is finitely generated and it is not difficult to find a minimal system of generators of $\Gamma$, see. e.g. Rosales and García Sánchez 7 .

The number $c(\Gamma)=\max (\mathbb{N} \backslash \Gamma)+1$ is called the conductor of $\Gamma$; in particular $c(\Gamma)-1$ is the Frobenius number of $\Gamma$. The computation of $c(\Gamma)$ for an arbitrary number of minimal generators of $\Gamma$ is NP-hard (see Ramírez Alfonsín [6] for a good account of this), but there are some special cases in which a closed formula is available. For example, if $\Gamma=\alpha \mathbb{N}+\beta \mathbb{N}:=\langle\alpha, \beta\rangle$, then $c(\Gamma)=\alpha \beta-\alpha-\beta+1$. However, for a numerical semigroup with more than two generators it is not possible in general to obtain a closed polynomial formula for its conductor in terms of the minimal set of generators (see Curtis [2]). We are interested in subsets of $\mathbb{N}$ which have an additive structure over $\Gamma$ (in analogy with the structure of module over a ring): a $\Gamma$-semimodule is a non-empty subset $\Delta$ of $\mathbb{N}$ with $\Delta+\Gamma \subseteq \Delta$. A system of generators of $\Delta$ is a subset $\mathcal{E}$ of $\Delta$ such that $\Delta=\bigcup_{x \in \mathcal{E}}(x+\Gamma)$; it is called minimal if no proper subset of $\mathcal{E}$ generates $\Delta$. Notice that, since $\Delta \backslash \Gamma$ is finite,

2010 Mathematics Subject Classification. Primary: 20M14; Secondary: 05A19.

Key words and phrases. Numerical semigroup, Frobenius problem, $\Gamma$-semimodule, syzygy.

The first author was partially supported by Spanish Goverment, Ministerios de Ciencia e Innovación y de Universidades MTM2016-76868-C2-1-P. The second author was partially supported by the Spanish Government, Ministerios de Ciencia e Innovación y de Universidades, grant PGC2018-096446-B-C22, as well as by Universitat Jaume I, grant UJI-B2018-10. 
every $\Gamma$-semimodule is finitely generated and has a conductor

$$
c(\Delta)=\max (\mathbb{N} \backslash \Delta)+1
$$

Motivated by the Frobenius problem, it is natural to ask for a closed formula for the conductor of a $\Gamma$-semimodule. The purpose of this note is to give a formula for $c(\Delta)$ in the case $\Gamma=\langle\alpha, \beta\rangle$ in terms of the generators of the semimodule of syzygies of $\Delta$, see [4], as well as in terms of the generators of the dual of this semimodule, see [5]. These are the contents of our two main results, namely Theorem 3.1 resp. Corollary 3.6.

\section{Semimodules over a Numerical SEmigroup}

Let $\Gamma$ be a numerical semigroup. This section is devoted to collect the main properties concerning $\Gamma$-semimodules. The reader is referred to [7] or [6] for specific material about numerical semigroups.

Every $\Gamma$-semimodule $\Delta$ has a unique minimal system of generators (see e.g. 4, Lemma 2.1]). Two $\Gamma$-semimodules $\Delta$ and $\Delta^{\prime}$ are called isomorphic if there is an integer $n$ such that $x \mapsto x+n$ is a bijection from $\Delta$ to $\Delta^{\prime}$; we write then $\Delta \cong \Delta^{\prime}$. For every $\Gamma$ semimodule $\Delta$ there is a unique semimodule $\Delta^{\prime} \cong \Delta$ containing 0 ; such a semimodule is called normalized. Moreover, the minimal system of generators $\left\{x_{0}=0, \ldots, x_{n}\right\}$ of a normalized $\Gamma$-semimodule is a $\Gamma$-lean set, i.e. it satisfies that

$$
\left|x_{i}-x_{j}\right| \notin \Gamma \text { for any } 0 \leq i<j \leq n,
$$

and conversely, every $\Gamma$-lean set of $\mathbb{N}$ minimally generates a normalized $\Gamma$-semimodule. Hence there is a bijection between the set of isomorphism classes of $\Gamma$-semimodules and the set of $\Gamma$-lean sets of $\mathbb{N}$. See Sect. 2 in [4] for the proofs of those statements.

There is another kind of system of generators - not minimal-for a semimodule $\Delta$ of $\Gamma$ relative to $s \in \Gamma \backslash\{0\}$ : this is the set of the $s$ smallest elements in $\Delta$ in each of the $s$ classes modulo $s$, namely the set $\Delta \backslash(s+\Delta)$, and is called the Apéry set of $\Delta$ with respect to $s$; we write $\operatorname{Ap}(\Delta, s)$.

A formula for the conductor in terms of $\operatorname{Ap}(\Delta, s)$ for $s \in \Gamma \backslash\{0\}$ is easily deduced.

Proposition 2.1. Let $\Delta$ be a $\Gamma$-semimodule. For any $s \in \Gamma \backslash\{0\}$ we have that

$$
c(\Delta)-1=\max _{\leq_{\mathbb{N}}} \operatorname{Ap}(\Delta, s)-s .
$$

Proof. The equality follows as in the case $\Delta=\Gamma$, see e.g. Lemma 3 in Brauer and Shockley [1].

In this paper we will consider numerical semigroups with two generators, say $\Gamma=\langle\alpha, \beta\rangle$, with $\alpha, \beta \in \mathbb{N}$ with $\alpha<\beta$ and $\operatorname{gcd}(\alpha, \beta)=1$. As mentioned above, the conductor of $\Gamma$ can be expressed as $c=c(\langle\alpha, \beta\rangle)=(\alpha-1)(\beta-1)$. The gaps of $\langle\alpha, \beta\rangle$ are also easy to describe: they admit a unique representation $\alpha \beta-a \alpha-b \beta$, where $a \in] 0, \beta-1] \cap \mathbb{N}$ and $b \in] 0, \alpha-1] \cap \mathbb{N}$. This writing yields a map from the set of gaps of $\langle\alpha, \beta\rangle$ to $\mathbb{N}^{2}$ given by

$$
\alpha \beta-a \alpha-b \beta \mapsto(a, b),
$$

which allows us to identify a gap with a lattice point in the lattice $\mathcal{L}=\mathbb{N}^{2}$; since the gaps are positive numbers, the point lies inside the triangle with vertices $(0,0),(0, \alpha),(\beta, 0)$. 


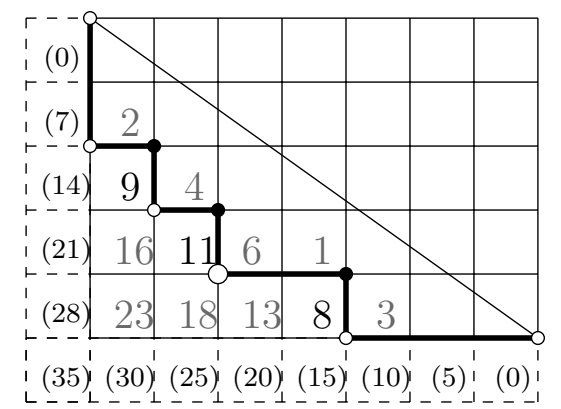

Figure 2.1. Lattice path for the $\langle 5,7\rangle$-lean set $I=[0,9,11,8]$ and the corresponding syzygy minimal generators $J=[14,16,18,15]$. The biggest generator $M$ with respect to $\leq_{\mathbb{N}}$ is depicted bigger.

In the following we will use the notation

$$
e=\alpha \beta-a(e) \alpha-b(e) \beta
$$

for a gap $e$ of the semigroup $\langle\alpha, \beta\rangle$; if the gap is subscripted as $e_{i}$ then we write $a_{i}=a\left(e_{i}\right)$ and $b_{i}=b\left(e_{i}\right)$.

Let us denote by $\leq$ the total ordering in $\mathbb{N}$; sometimes we will write $\leq_{\mathbb{N}}$ to emphasize that it is the natural ordering. In addition, we define the following partial ordering $\preceq$ on the set of gaps:

Definition 2.2. Given two gaps $e_{1}, e_{2}$ of $\langle\alpha, \beta\rangle$, we define

$$
e_{1} \preceq e_{2}: \Longleftrightarrow a_{1} \leq a_{2} \wedge b_{1} \geq b_{2}
$$

and

$$
e_{1} \prec e_{2}: \Longleftrightarrow a_{1}<a_{2} \wedge b_{1}>b_{2} .
$$

Observe that the ordering $\preceq$ differs from the one used by the second author and Uliczka in [3, 4, 5]: there the gaps $e_{i}$ are ordered by decreasing sequence of the corresponding $a_{i}$. Let $\mathcal{E}=\left\{0, e_{1}, \ldots, e_{n}\right\} \subseteq \mathbb{N}$ with gaps $e_{i}=\alpha \beta-a_{i} \alpha-b_{i} \beta$ of $\langle\alpha, \beta\rangle$ for every $i=1, \ldots, n$ such that $a_{1}<a_{2}<\cdots<a_{n}$. Corollary 3.3 in [4] ensures that $\mathcal{E}$ is $\langle\alpha, \beta\rangle$-lean if and only if $b_{1}>b_{2}>\cdots>b_{n}$.

This simple fact leads to an identification (cf. [4, Lemma 3.4]) between an $\langle\alpha, \beta\rangle$-lean set and a lattice path with steps downwards and to the right from $(0, \alpha)$ to $(\beta, 0)$ not crossing the line joining these two points, where the lattice points identified with the gaps in $\mathcal{E}$ mark the turns from the $x$-direction to the $y$-direction; these turns will be called ES-turns for abbreviation. Figure 2.1 shows the lattice path corresponding to the $\langle 5,7\rangle$-lean set $\{0,9,11,8\}$.

Let $g_{0}=0, g_{1}, \ldots, g_{n}$ be the minimal system of generators of a $\langle\alpha, \beta\rangle$-semimodule $\Delta$. From now on, we will assume that the indexing in the minimal set of generators of $\Delta$ is such that $g_{0}=0 \preceq g_{1} \preceq \cdots \preceq g_{n}$; accordingly we will use the notation $\left[g_{0}, \ldots, g_{n}\right]$ rather than $\left\{g_{0}, \ldots, g_{n}\right\}$. In [4] it was introduced the notion of syzygy of $\Delta$ as the $\langle\alpha, \beta\rangle$-semimodule

$$
\operatorname{Syz}(\Delta):=\bigcup_{i, j \in\{0, \ldots n\}, i \neq j}\left(\left(\Gamma+g_{i}\right) \cap\left(\Gamma+g_{j}\right)\right) .
$$


The semimodule of syzygies of the semimodule $\Delta$ minimally generated by $\left[g_{0}=0, g_{1}, \ldots, g_{n}\right]$ can be characterized as follows (see [4, Theorem 4.2]; since Definition 2.2 differs from the corresponding in [3, 4, 5] - as mentioned above, Definition 2.3 must be conveniently adapted here):

\section{Definition 2.3.}

$$
\operatorname{Syz}(\Delta)=\bigcup_{0 \leq k<j \leq n}\left(\left(\Gamma+g_{k}\right) \cap\left(\Gamma+g_{j}\right)\right)=\bigcup_{k=0}^{n}\left(\Gamma+h_{k}\right),
$$

where $h_{1}, \ldots, h_{n-1}$ are gaps of $\Gamma, h_{0}, h_{n} \leq \alpha \beta$, and

$$
\begin{aligned}
& h_{k} \equiv g_{k} \bmod \beta, h_{k}>g_{k} \text { for } k=0, \ldots, n \\
& h_{k} \equiv g_{k+1} \bmod \alpha, h_{k}>g_{k+1} \text { for } k=0, \ldots, n-1 \\
& h_{n} \equiv 0 \bmod \alpha, \text { and } h_{n} \geq 0
\end{aligned}
$$

In particular, $J=\left[h_{0}, \ldots, h_{n}\right]$ is a minimal system of generators of the semimodule $\operatorname{Syz}(\Delta)$, hence $h_{0} \preceq h_{1} \preceq \cdots \preceq h_{n}$. Therefore it is easily seen that the SE-turns of the lattice path associated to $\Delta$ can be identified with the minimal set of generators of the syzygy module (we call SE-turns to the turns from the $y$-direction to the $x$-direction). After that, we can associate to any $\Gamma$-semimodule $\Delta$ a lean set $[I, J]$, where $I$ is a minimal set of generators of $\Delta$ and $J$ a minimal set of generators of $\operatorname{Syz}(\Delta)$; or, equivalently, a lattice path. An easy consequence of this fact is the following lemma.

Lemma 2.4. Let $\Delta$ be a $\Gamma$-semimodule with associated $\Gamma$-lean set $[I, J]$ for $I=\left[g_{0}=\right.$ $\left.0, g_{1}, \ldots, g_{n}\right]$ and $J=\left[h_{0}, \ldots, h_{n}\right]$. Then, for any $h \in J$ we have $h-\alpha-\beta \notin \Delta$.

Proof. Consider $h \in J$ such that that $g_{i} \prec h \prec g_{i+1}$. Let us denote $\left(a_{j}, b_{j}\right)$ resp. $\left(a_{j+1}, b_{j+1}\right)$ the coordinates of $g_{j}$ resp. $g_{j+1}$ in the lattice $\mathcal{L}$; then the element $h$ is represented in the lattice path as $\left(a_{j}, b_{j+1}\right)$, see Definition 2.3. By contradiction, assume that $h-\alpha-\beta \in \Delta$; then there exists a gap $g \in I$ together with two integers $\nu_{1}, \nu_{2} \in \mathbb{N}$ such that

$$
h-\alpha-\beta=\nu_{1} \alpha+\nu_{2} \beta+g .
$$

Since $h-\alpha-\beta \notin \Gamma$, we may write

$$
h-\alpha-\beta=\alpha \beta-\left(a_{j}+1\right) \alpha-\left(b_{j+1}+1\right) \beta .
$$

The writing of $g$ as $g=\alpha \beta-a \alpha-b \beta$ is unique whenever $(a, b) \in \mathcal{L}$, therefore

$$
a_{j}+1=a-\nu_{1}, \quad b_{j+1}+1=b-\nu_{2} .
$$

These equalities yield the conditions $a_{j}<a$ and $b_{j+1}<b$. But the unique minimal generator which fulfills these conditions is $g_{j+1}$; however, $h$ cannot be expressed as $h=$ $g_{j+1}+\nu+\alpha+\beta$ since $h$ is represented in the lattice path as $\left(a_{j}, b_{j+1}\right)$, a contradiction.

Example 2.5. For $\Gamma=\langle 5,7\rangle$ and the $\Gamma$-semimodule $\Delta_{I}$ minimally generated by $I=$ $[0,9,11,8]$, it is easily deduced that the syzygy module $\operatorname{Syz}\left(\Delta_{I}\right)$ is minimally generated by $J=[14,16,18,15]$, cf. Figure [2.1; there we have extended the labelling beyond the axis in the natural way in order to have also an interpretation of $J$ in terms of the lattice path. Observe that by Lemma 2.4 we have $14-7-5=2 \notin \Delta, 16-7-5=4 \notin \Delta$, $18-7-5=6 \notin \Delta$ and $15-7-5=3 \notin \Delta$; this can be read off from Figure 2.1 as well. 


\section{A FORMUlA FOR THE CONDUCTOR OF AN $\langle\alpha, \beta\rangle$-SEMimodule}

In this section we are going to provide a formula for the conductor of a $\Gamma$-semimodule with any number of generators in terms of the generators of $\Gamma$ and a special syzygy of the $\Gamma$-semimodule. In particular, we will obtain some relations between the conductor of $\Gamma$ and the conductor of the $\Gamma$-semimodule. Finally, we will provide a formula for the conductor of the $\Gamma$-semimodule in terms of its dual.

Theorem 3.1. Let $\Delta$ be a $\Gamma$-semimodule with associated lean set $[I, J]$ as above, and let $M:=\max _{\leq_{\mathbb{N}}}\{h \in J\}$ denote the biggest (with respect to the total ordering of the natural numbers) minimal generator of $\operatorname{Syz}(\Delta)$. Then

$$
c(\Delta)=M-\alpha-\beta+1 \text {. }
$$

In particular, if $\left(m_{1}, m_{2}\right)$ are the coordinates of the point representing $M$ in the lattice $\mathcal{L}$, then we have

$$
c(\Delta)=c(\Gamma)-m_{1} \alpha-m_{2} \beta .
$$

Proof. Since $c(\Delta)-1$ is the Frobenius number of the $\Gamma$-semimodule $\Delta$, it is enough to check that (i) $M-\alpha-\beta \notin \Delta$, and (ii) if $\ell \notin \Delta$, then $\ell \leq M-\alpha-\beta$. The statement (i) is clear by Lemma 2.4, since $M \in J$. To see (ii), consider an element $\ell \notin \Delta$, which in particular means $\ell \notin \Gamma$. So we can associate to $\ell$ a point $(a, b)$ in the lattice $\mathcal{L}$. Moreover, $\ell$ is upon and not contained in the lattice path associated to $I$. This means that there exists some $j \in J$ with coordinates $\left(j_{1}, j_{2}\right)$ in the lattice path such that $a>j_{1}$ and $b>j_{2}$, otherwise $\ell$ would be an element of $\Delta$, since the elements represented by lattice points on and under the lattice path belong to $\Delta$. Therefore, $a \geq j_{1}+1$ and $b \geq j_{2}+1$. Thus, from the representation of $\ell$ and $j$ as gaps we can check that

$$
\ell=\alpha \beta-a \alpha-b \beta \leq_{\mathbb{N}} \alpha \beta-\left(j_{1}+1\right) \alpha-\left(j_{2}+1\right) \beta=j-\alpha-\beta .
$$

Hence, since $M=\max _{\leq_{\mathbb{N}}}\{h \in J\}$ and $M \in J$, we have that $M-\alpha-\beta \geq_{\mathbb{N}} \ell$ for any $\ell \notin \Delta$, which proves (ii).

Finally, since $M$ can be represented as a lattice point $\left(m_{1}, m_{2}\right) \in \mathcal{L}$, we have

$$
c(\Delta)=M-\alpha-\beta+1=\alpha \beta-m_{1} \alpha-m_{2} \beta-\alpha-\beta+1=c(\Gamma)-m_{1} \alpha-m_{2} \beta .
$$

Example 3.2. Again in the case of $\Gamma=\langle 5,7\rangle$ and the $\Gamma$-semimodule minimally generated by $[0,9,11,8]$, Figure 2.1 illustrates that the maximal syzygy is $M=18$, and so the conductor of the semimodule is $c(\Gamma)-5 m_{1}-7 m_{2}=24-5 \cdot 2-7 \cdot 1=7$.

Notice that for the particular case of $\Delta=\Gamma$ we have $M=\alpha \beta$, and we recover the wellknown formula $c(\Gamma)=\alpha \beta-\alpha-\beta+1$. The value $M$ can be easily characterized in terms of the Apéry set of $\Delta$ with respect to $\alpha+\beta$ :

Proposition 3.3. Let $M:=\max _{\leq_{\mathbb{N}}}\{h \in J\}$ be the biggest minimal generator of the syzygy module with respect to the natural ordering of $\mathbb{N}$ as above, then

$$
M=\max _{\leq \mathbb{N}} \operatorname{Ap}(\Delta, \alpha+\beta) .
$$

Proof. This is a consequence of Proposition 2.1 for $s=\alpha+\beta \in\langle\alpha, \beta\rangle$. 
A straightforward consequence of Theorem 3.1 is the following.

Corollary 3.4. Let $\Delta$ be a $\Gamma$ semimodule. Then

$$
c(\Gamma)-c(\Delta) \in \Gamma \text {. }
$$

We conclude this paper rewriting the formula of Theorem 3.1 in terms of the dual $\Gamma-$ semimodule of $\Delta$,

$$
\Delta^{*}:=\{z \in \mathbb{Z} \mid z+\Delta \subset \Gamma\}
$$

see [5]. An important fact about the dual semimodule is that the minimal set of generators of $\operatorname{Syz}(\Delta)$ is in bijection with the minimal set of generators of $\Delta^{*}$ :

Lemma 3.5 ([5], Lemma 6.1). The minimal sets of generators of $\Delta^{*}$ and $\operatorname{Syz}(\Delta)$ are in correspondence via the map $x \mapsto \alpha \beta-x$.

In particular, this bijection together with Theorem 3.1 allows us to compute the conductor of the semimodule $\Delta$ in terms of the minimal generators of $\Delta^{*}$ in a natural way:

Corollary 3.6. Let $\Delta$ be a $\Gamma$-semimodule, and let $\Delta^{*}$ be its dual, minimally generated by $x_{0}, \ldots, x_{n}$. Then

$$
c(\Delta)=\alpha \beta-\min _{\leq \mathbb{N}}\left\{x_{0}, \ldots, x_{n}\right\}-\alpha-\beta+1 .
$$

Proof. By Theorem 3.1 we have that $c(\Delta)=\max _{\leq_{\mathbb{N}}}\{h \in J\}-\alpha-\beta+1$, where $J$ is a minimal set of generators of $\operatorname{Syz}(\Delta)$. Lemma 3.5 yields the equality

$$
\min _{\leq \mathbb{N}}\left\{x_{0}, x_{1}, \ldots, x_{n}\right\}=\alpha \beta-\max _{\leq \mathbb{N}}\{h \in J\},
$$

which allows us to conclude.

Example 3.7. By [5, Theorem 2.5], the minimal generators of the dual of the $\langle 5,7\rangle$ semimodule $\Delta_{I}$ are given by $[20,17,19,21]$; notice that, for the explicit calculation, the mentioned theorem requires the reverse ordering $\succeq$ instead of the ordering $\preceq$ we use here. The minimum of this set is 17 , therefore by Corollary 3.6 we have $c(\Delta)=35-17-12+1=$ 7, as computed in Example 3.2 .

\section{REFERENCES}

1. A. Brauer, J.E. Shockley, On a problem of Frobenius, J. reine und angewandte Math. 211 (1962), $215-220$.

2. F. Curtis, On formulas for the Frobenius number of a numerical semigroup, Math. Scand. 67, no. 2 (1990), 190-192.

3. J.J. Moyano-Fernández, J. Uliczka, Hilbert depth of graded modules over polynomial rings in two variables, J. Algebra 373 (2013), 130-152.

4. J.J. Moyano-Fernández, J. Uliczka, Lattice paths with given number of turns and numerical semigroups, Sem. Forum 88, no. 3, (2014), 631-646.

5. J.J. Moyano-Fernández, J. Uliczka, Duality and syzygies for semimodules over numerical semigroups, Sem. Forum 92, no. 3, (2016), 675-690.

6. J. L. Ramírez Alfonsín, The Diophantine Frobenius problem, Oxford Lecture Series in Mathematics and its Applications 30, Oxford University Press, Oxford (2005)

7. J. C. Rosales, P. A. García Sanchez, Numerical Semigroups, Springer, New York (2009). 
A FORMULA FOR THE CONDUCTOR OF A SEMIMODULE OF SOME NUMERICAL SEMIGROUPS7

Instituto de Matemática interdisciplinar (IMI) y departamento de Álgebra, Geometría y Topología, Facultad de Ciencias Matemáticas, Universidad Complutense de Madrid, 28040, MADRID, SPAIN.

Email address: palmiron@ucm.es

Universitat Jaume I, Campus de Riu Sec, Departamento de Matemáticas \& Institut Universitari de Matemàtiques i Aplicacions de Castelló, 12071 Castellón de la Plana, Spain Email address: moyano@uji.es 